\title{
Solução das Equações de Cinética Pontual de nêutrons via série de Potências
}

\author{
Fernanda Tumelero* Claudio Z. Petersen Glênio A. Gonçalves \\ Universidade Federal de Pelotas / IFM / DME \\ Campus Universitário, $\mathrm{s} / \mathrm{n}^{\circ}$. Capão do Leão, RS \\ Emails: fernanda.tumelero@yahoo.com.br; claudio.petersen@ufpel.edu.br; glenio_a@yahoo.com.
}

\section{RESUMO}

As equações de cinética pontual de nêutrons na dinâmica de um reator nuclear consistem em um sistema acoplado de equações diferenciais ordinárias. Uma importante característica das equações de cinética é ser um sistema do tipo rígido. Essas equações envolvem exclusivamente a variação da amplitude do fluxo com o tempo, ou seja, assumem total separabilidade no tempo e no espaço, na qual a forma espacial do fluxo é conhecida o que torna essas equações exclusivamente dependentes do tempo. O objetivo deste trabalho consiste na solução das equações de cinética pontual de nêutrons considerando o modelo com 6 grupos de precursores de nêutrons atrasados para reatividades do tipo: rampa, zig-zag e senoidal. Resolve-se este problema fazendo uma aproximação linear local via série de potências em conjunto com a continuação analítica. Um controle de erro local é realizado via Estimador de Lagrange e os resultados obtidos são comparados com aqueles encontrados na literatura.

As equações de cinética pontual de nêutrons são dadas por:

$$
\begin{aligned}
& \frac{d n(t)}{d t}=\frac{\rho(t)-\beta}{\Lambda} n(t)+\sum_{i} \lambda_{i} c_{i}(t) \\
& \frac{d c_{i}(t)}{d t}=\frac{\beta_{i}}{\Lambda} n(t)-\lambda_{i} c_{i}(t)
\end{aligned}
$$

onde $i=1,2, \ldots, M$ (com $\mathrm{M}$ sendo o $\mathrm{n}^{\mathrm{o}}$ de precursores). Com as seguintes condições iniciais:

$$
\begin{aligned}
& n(0)=1 \\
& C_{i}(0)=\frac{\beta_{i}}{\lambda_{i} \Lambda}, \quad i=1,2, \ldots 6
\end{aligned}
$$

onde $n(t)$ é a densidade de nêutrons, $\rho(t)$ é a reatividade, $\beta$ é a fração total de nêutrons atrasados, $\Lambda$ é o tempo médio de geração de nêutrons, $\lambda_{i}$ é a constante de decaimento no grupo $i$ de precursores, $\beta_{i}$ é a fração de nêutrons atrasados no grupo $i$ de precursores e $C_{i}(t)$ é a concentração de nêutrons atrasados no grupo $i$ de precursores.

A ideia é encontrar uma solução para o sistema (1) em forma de séries de potências em torno de um ponto ordinário $t_{0}$, procurando uma solução da forma:

$$
\begin{aligned}
& n(t)=a_{0}+a_{1}\left(t-t_{0}\right)+\ldots+a_{n}\left(t-t_{0}\right)^{n}=\sum_{n=0}^{\infty} a_{n}\left(t-t_{0}\right)^{n} \\
& C_{i}(t)=b_{i, 0}+b_{i, 1}\left(t-t_{0}\right)+\ldots+b_{i, n}\left(t-t_{0}\right)^{n}=\sum_{n=0}^{\infty} b_{i, n}\left(t-t_{0}\right)^{n}
\end{aligned}
$$

Substituindo (3) e sua derivada em (1) chega-se na relação de recorrência, que na forma explícita é expressa por: 


$$
a_{n+1}=\frac{\frac{\rho(t)-\beta}{\Lambda} a_{n}+\lambda b_{i, n}}{(n+1)} ; \quad b_{i, n+1}=\frac{\frac{\beta}{\Lambda} a_{n}-\lambda b_{i, n}}{(n+1)}
$$

Através das condições iniciais pode-se determinar $a_{0}$ e $b_{0}$ iniciando a geração dos $a_{n}$ e $b_{n}$. Primeiramente procura-se soluções em séries em torno de um ponto $t_{0}$ num intervalo $I_{0}=[0,2 \Delta t]$, onde $\Delta t=t_{0}$ é o passo de tempo escolhido. Admitindo $\rho(t)=\rho$ e que a densidade de nêutrons e a concentração de precursores são uma aproximação linear local em torno de $t_{0}$ para $I_{0}$, temos que a série (3) torna-se:

$$
\begin{aligned}
& n(t) \cong a_{0}+a_{1}\left(t-t_{0}\right) \\
& C_{i}(t) \cong b_{i, 0}+b_{i, 1}\left(t-t_{0}\right)
\end{aligned}
$$

Agora, utilizando a mesma ideia para todos os intervalos $I_{n}=[(2 n) \Delta t,(2 n+2) \Delta t]$ para $\mathrm{n}=0,1,2, \ldots$, somos capazes de encontrar a solução para todos os intervalos $I_{n+1}$ em torno de $t_{0}$ pertencente a esse intervalo tomando como condição inicial a solução no intervalo anterior $I_{n} \mathrm{em}$ $t=(n+2) \Delta t$ para $\mathrm{n}=0,1,2 \ldots$, ou seja, fazendo uso da continuação analítica.

Para efeito de ilustração mostra-se os resultados obtidos para o caso de reatividade tipo rampa $(0.1 \$ / \mathrm{s})$ comparados com [3], para isso, assume-se os seguintes parâmetros: $\Lambda=0.00003$, $\beta=0.006473, \beta_{l}=(0.000214,0.001423,0.001247,0.002568,0.000748,0.000273)$ e $\lambda_{l}=(0.0124$, $0.0305,0.111,0.301,1.14,3.01)$.

\begin{tabular}{cccc}
\hline$t(s)$ & Série de Potências $\Delta t=0.0001$ & PCA $^{[3]}$ & EPCA $^{[3]}$ \\
\hline 2 & 1.33820005 & 1.338200434 & 1.33820005 \\
4 & 2.228441895 & 2.228442526 & 2.228441897 \\
6 & 5.582052438 & 5.582053977 & 5.58205245 \\
8 & 42.78629544 & 42.78630638 & 42.78629574 \\
10 & 451164.0975 & 451163.6225 & 451163.624 \\
11 & $17.92327126^{*} 10^{\wedge} 15$ & $17.92212523^{*} 10^{\wedge} 15$ & $17.2213608^{*} 10^{\wedge} 15$ \\
\hline \multicolumn{4}{c}{}
\end{tabular}

O método de séries de potências se comporta de maneira semelhante a polinômios e são fáceis de manipular analiticamente e numericamente. Em todos os casos para diferentes reatividades, o método apresentou uma excelente precisão comparado aos resultados presentes na literatura, considerando que realizou-se uma aproximação linear.

Palavras-chave: Cinética Pontual de nêutrons, Reatores Nucleares, Séries de Potência.

\section{Referências}

[1] Petersen, C. Z.; Dulla, S.; Vilhena, M. T.; Ravetto, R. An analytical solution of the point kinetics equations with time-variable reactivity by the decomposition method, Progress in Nuclear Energy (New series), p. 1-4, 2011 a.

[2] Duderstadt, J. J.; Hamilton, L. J. Nuclear Reactor Analysis, John Wiley \& Sons, New York, 1976.

[3] P. Picca; R. Furfaro; B.D. Ganapol, A highly accurate technique for the solution of the nonlinear point kinetics equations. Nuclear Energy, vol. 58, pp.43-53, (2012). 